\title{
Clinical decision making in postmastectomy radiotherapy in node negative breast cancer
}

\author{
Rodrigo Barrientos ${ }^{1}$, Suraj Samtani ${ }^{3}$, Michael Frelinghuysen ${ }^{2}$, Camilo Sotomayor ${ }^{2}$, Juan Guillermo Gormaz ${ }^{3}$ and \\ Mauricio Burotto ${ }^{4}$
}

1Department of Radiation Oncology, Instituto de Radiomedicina IRAM, Santiago, Chile

${ }^{2}$ Radiation Oncology Unit, Hospital Clinico Regional de Concepción, Concepción, Chile

${ }^{3}$ Department of Medical Oncology, Clinica Alemana Santiago, Vitacura, Chile

${ }^{4}$ Centro de Investigación Clínica Bradfordhill, Santiago, Chile

Correspondence to: Mauricio Burotto. Email: mauricioburotto@gmail.com

\begin{abstract}
For decades, postmastectomy radiotherapy (PMRT) has been recommended for node positive $[\mathrm{N}(+)]$ breast cancer patients; nevertheless, the beneficial effect of PMRT for treatment of node negative $[\mathrm{N}(-)]$ disease remains under discussion. Nowadays, the biology of breast cancer and the risk factors (RFs) for locoregional failure (LRF) must be included in the decision on whether or not to carry out PMRT. For these reasons, the present review aims to evaluate the rationale use of PMRT in $\mathrm{N}(-)$ patients and discuss which subgroups may further benefit from the treatment in present times where the decision must be personalised, according to the RFs of locoregional recurrence (LRR). To perform the analysis, we ponder that LRR of over $10 \%$ should be considered unacceptable due to the fact that LRRs generate great morbidity in patients. For this purpose, we consider that routine RT in these patients is not recommended, although there are subgroups of patients with high LRR, in which PMRT could be beneficial.
\end{abstract}

Keywords: postmastectomy radiotherapy, node negative breast cancer, breast cancer radiotherapy

Published: 26/09/2018

Received: 25/04/2018

ecancer 2018, 12:874 https://doi.org/10.3332/ecancer.2018.874

Copyright: $\odot$ the authors; licensee ecancermedicalscience. This is an Open Access article distributed under the terms of the Creative Commons Attribution License (http://creativecommons.org/licenses/by/3.0), which permits unrestricted use, distribution, and reproduction in any medium, provided the original work is properly cited. 


\section{Introduction}

Currently, breast cancer is the second most prevalent cancer in women, after nonmelanoma skin malignant diseases. In fact, it is estimated that $30 \%$ of new cancer cases and $25 \%$ of deaths caused by cancer in women are due to breast cancer in 2018 [1], with a cumulative incidence and mortality of $126.01 / 100,000$ and $27.91 / 100,000$, respectively [2, 3].

For decades, postmastectomy radiotherapy (PMRT) has been recommended for node positive [N(+)] breast cancer patients. Of note, between 1997 and 2005, three randomised controlled trials reignited the debate about the role of PMRT, especially in the specific clinical setting of $\mathrm{N}(+)$ disease patients [4-6]; however, the beneficial effects of PMRT for treatment of node negative [N(-)] disease remains under discussion [7].

The present review has the purpose of evaluating the indications of PMRT in N(-) patients and discusses which subgroups may further benefit from treatment in this setting. To analyse this issue, we ponder that locoregional recurrence (LRR) of over $10 \%$ should be considered unacceptable, based on node negative breast conservative randomised trials that showed LRR rates of $6.2 \%-6.7 \%$ [8, 9].

\section{Role of postmastectomy radiotherapy}

A recent meta-analysis from the Early Breast Cancer Trialists' Collaborative Group (EBCTCG) [7] investigated 22 trials that included 8,135 breast cancer patients who were treated with total mastectomy (TM), randomised to receive either further RT or observation. At 20 years of follow-up, $\mathrm{N}(+)$ disease patients who received PMRT showed a reduction in risk of LRR from $26 \%$ to $8.1 \%$, with a reduction in absolute risk of breast cancer mortality of $8.1 \%$ (RR $0.84 ; 95 \% \mathrm{Cl} 0.76-0.94)$, whereas PMRT in $\mathrm{N}(-)$ disease patients increased overall mortality (RR $1.23 ; 95 \% \mathrm{Cl} 1.02-1.49,2 p=0.03)$, despite the fact that current numbers show that mortality in N0 patients is low.

Locoregional relapse should always be considered for the analysis of PMRT effectiveness because radiation therapy exerts its main benefit decreasing its occurrence. In the context of mastectomised patients, LRR is associated with a diminished quality of life, higher risk of mortality and lack of satisfactory results after performing either surgical or radiation rescue therapy. To clarify the magnitude of this problem, once an LRR has been diagnosed, patients have decreased survival rates (ranging from 46\% to 63\%) [10-12] and higher risk of distant failure (DF) [13]. In fact, Locoregional control plays an important role in both overall survival (OS) and distant disease [10, 11, 13, 14]. The time of diagnosis is especially relevant considering that adjuvant chemotherapy (QT) has shown poor results and no OS benefit after isolated LRR [15-18].

For all these reasons, and the fact that rational use of irradiation according to normal tissue tolerance doses is rarely associated with acute skin moist desquamation and late adverse effects (such as rib fracture, radiation pneumonitis, ischaemic heart disease and second malignancies) [19-22], we think that certain subgroups of N0 patients would benefit from receiving PMRT.

\section{Why PMRT may benefit different subgroups of $\mathrm{N}(-)$ patients?}

Breast cancer is a heterogeneous disease with several molecular subtypes. Perou et al classified breast cancer into four distinct molecular subtypes based on a genetic profile: Luminal A, Luminal B, Her2 enriched and basal-like [23, 24], each of them with variable prognosis and different survival rates. In fact, Triple Negative Breast Cancer (TNBC) (a surrogate of basal-like disease) is associated with an increased risk of LRR [25, 26] and breast cancer specific survival (BCSS) [27]. This continues to be a topic under active investigation, but a proper analysis of the data can help in taking a proper decision regarding whether a patient could benefit from PMRT.

As a matter of fact, RT seems to have a role in the adjuvant management of T1-3N0 patients who have received mastectomy when molecular subtypes are considered; however, these benefits are still not fully understood [28, 29]. Moreover, consideration of genetic profiles in clinical practice is associated with technical and economic limitations, especially in developing countries. The OncotypeDX recurrence score can predict LRR in breast cancer patients [30]; however, there is currently no clear evidence that can assist in decision making for adjuvant RT. Thus, up to this date, clinical-pathologic biomarkers are still used as a surrogate for genetic profile. 
Some proven clinical-pathological factors that influence local control in breast cancer are age, tumour size, lymphovascular invasion (LVI), histological grade and no use of systemic QT [31-35]. Therefore, since RT exerts benefits, particularly in LRR, one could expect that PMRT could play a role in patients who present these factors as they are at higher risk of LRR.

\section{Radiation therapy in pathologic stage T1-T2 N0 patients}

There is no clear evidence that T1-2N0 breast cancer patients may benefit from PMRT. The incidence of LRR is low and many patients at risk of failure would need to be treated in order demonstrate a benefit.

Two independent data sets were pooled and analysed by Truong et al [28]. At a median follow-up of 4.3 years, they reported on 1994 mastectomised breast cancer patients, treated without adjuvant RT between 1998 and 2009, LRR rates of $1.8 \%, 3.1 \%, 1.7 \%, 1.9 \%$ and $1.9 \%$ in luminal A, luminal B, Her2 (+) luminal, Her2 (+) non-luminal and TNBC breast cancer patients, respectively. Multivariate analyses reported higher risk of LRR for tumours $>2 \mathrm{~cm}$, lobular histology and presence of close or positive margins. Thus, these factors should be considered along with diverse molecular factors to ponder the risk of LRR after PMRT. Nevertheless, it is important to take into account that systemic therapy was less commonly used in TNBC patients compared to other subtypes of breast cancer $(p<0.001)$, which might have influenced the results.

In the same way, Abdulkarim et al [29] analysed 768 patients, T1-T3 N0-1 TNBC patients treated with breast conservative treatment (BCT), modified radical mastectomy (MRM) without RT and MRM plus RT with a median follow-up of 7.2 years. The multivariate analysis showed that both LVI and lymph node positivity $(\mathrm{N}+)$ were associated with an increased risk of LRR. In this population, 468 patients with T1-2N0 tumours with similar clinic pathological features were treated with BCT ( $n=233$ ) or MRM without RT $(n=235)$. Five-year LRR-free survival for T1-2N0 was $96 \%$ and $90 \%$ in the BCT and MRM groups, respectively $(p=0.022)$. The Cox multivariate analysis showed that MRM without RT was the only independent prognostic factor associated with increased risk of LRR in patients with TNBC, compared to BCT (HR 2.53; 95\% Cl 1.12-5.75, $p=0.02$ ). Adjuvant QT was not significantly associated with decreased risk of LRR.

From a study that investigated 390 mastectomies TNBC patients, including 307 T1-2 N0-1 patients, Chen et al [36] reported an LRR rate of $7.9 \%$. This study suggests that after radical mastectomy, TNBC tumours have low LRR and maybe other clinical and biological factors, besides receptor expression, may influence locoregional control.

In order to determine which subgroup of patients have a high risk of LRR, Emin Yildirim et al reported on 502 patients T1-2N0 treated with TM plus axillary dissection enrolled between 1990 and 2004 an LRR rate of $2.8 \%$, at a median follow-up of 77 months. The authors observed an inverse relationship between disease free survival (DFS) and tumour size. They also found that LVI positive patients had $13.2 \%$ of LR, compared to $1.2 \%$ in LVI negative patients [37]. For the analysis, they created two groups of patients: patients $<40$ years and patients $>40$ years old. Considering this subgroup analysis, they established at 10 years, that younger patients ( $<40$ years) with $\leq 1 \mathrm{RF}$ (tumour size and LVI) had an LRR free survival (LRRFS) of $98 \%$, compared to $44 \%$ in those patients with $>1 \mathrm{LRR}$ RF (HR 2.2; 95\% Cl 1.2-3.2, $p<0.0001)$ (Table 1), and established too that patients older than 40 years with $0-2$ factors had a $99 \%$ LRRF rate with respect to $57 \%$ in patients with three RFs (LVI, histological grade and tumour size) at 10 years (HR 2.0; 95\% Cl 1.3-2.8, $p<0.0001)$. Again, these data suggest that several factors must be considered when these types of patients are evaluated [37]. Likewise, Abi-Raad et al [34] analysed 1,136 patients T1-2N0 who have undergone mastectomy at a median follow-up of 9 years. They reported an LRR of $3.5 \%$ with a cumulative incidence of $5.2 \%$. In a multivariate analysis, factors associated with an increased risk of LRR were LVI $(p=0.002)$, positive margins $(p<0.001)$, tumour size $>2 \mathrm{~cm}(p=0.011)$, age $<50(p=0.018)$ and no use of systemic therapy $(p=0.036)$. The 10-year cumulative incidence of LRR in absence of any of these factors was $2 \%$, increasing to $3.3 \%$ with one RF, to $5.8 \%$ with two RFs and $19.7 \%(95 \% \mathrm{Cl} 12.2-28.6 \%, p<0.01)$ when three or more RFs were present.

These data were consistent with the results found by Jwa et al [38]; they analysed 390 T1-2N0-1 patients of which 307 were N0 patients. The factors associated with higher LRR in N0 patients at multivariate analysis were no use of adjuvant QT (HR 10.2; 95\% CI 1.2-88.5, $p=0.04)$ and age $<50$ years (HR 11.4; 95\% Cl 2.4-55.4, $p=0.01)$. 
Another study, which aimed to evaluate which subsets of patients have a higher risk of LRR to benefit from PMRT, was carried out by Trovo et al [39]. Based on 150 T1-2N0-1a breast cancer patients, who were treated with radical mastectomy without adjuvant RT, they reported in the univariate analysis that premenopausal status $(p=0.004)$, presence of $\mathrm{LVI}$, estrogen receptor (ER) negative $(p=0.02)$ and pathologic grade 3 tumour $(p=0.002)$ were associated with higher risk of LRR. In patients with these factors, the rates of LRR were $1.2 \%, 10.3 \%$, $24.1 \%$ and $75 \%$ if one, two, three or four of these factors were present, respectively $(p<0.001)$.

To identify a subgroup of patients at higher risk of LRR who could benefit from PMRT, Troung et al [40] analysed 1,505 women treated with MRM over 10 years between 1989 and 1999. A recursive partitioning analysis was used, and LRR rates over $20 \%$ were reported in patients who presented LVI plus high tumoural grade, and in patients with tumour size $\geq 2 \mathrm{~cm}$ plus high-grade histology. Age and size were also evaluated in a study involving 1,019 T1-2N0-1 patients when 753 were N(-). Sharma et al [41] reported an LRR rate of $2.3 \%$, with 10 years LRR rate in node negative patients of $10.5 \%$, for patients with age < 40 ; meanwhile patients $>40$ years had LRR rates of $1 \%$ $(p<0.0001)$. Among the 18 patients $\leq 40$ years with T2NO disease, the 10 -year LRR rates were $18.6 \%$, with younger age as the only significant independent predictor of LRR (HR 2.14; 95\% Cl 1.28-3.56, $P=0.004)$.

As per meta-analysis, close and positive margins are considered RFs for LRR in noninflammatory breast cancer [42]. Hastings et al [43] investigated 1,235 (1,259 cases) T1N0 patients who did not receive PMRT. With a median follow-up of 8.5 years, they observed a 10-year Kaplan-Meier LRR of $3.2 \%$ for all patients. Of note, the most common site of LRR was the chest wall (68\%). In the multivariate analysis, two factors were significantly associated with higher LRR. These were the margins $\leq 3 \mathrm{~mm}(\mathrm{HR} 2.97 ; 95 \% \mathrm{Cl} 1.21-7.29, p=0.02)$ and G3 (HR 3.97; 95\% Cl 1.94-8.14, $P=0.0002$ ). They also found that by combining these factors the 10-year Kaplan-Meier risk was $25 \%$ compared to $2.7 \%$ without them $(p<0.0001)$.

Ki 67, a nuclear protein present in cycling cells, is used as an indicator of tumour proliferation [44, 45], which has now been associated with worse DFS and OS [46]. Although there is not much literature that supports Ki67 as a single factor associated with a higher LRR risk, only one study has evaluated it as an RF for LRR and the role of PMRT. Selz et al [47] analysed 699 postmastectomy pN0 patients treated between 2001 and 1998 (191 patients with PMRT and 508 without PMRT), in order to establish the role of PMRT in LRR at a median followup of 56 months; they found only a Ki67 > 20\% was an independent factor for LRR (HR 4.18; 95\% Cl 1.11-15.77, $p<0.0215$ ).

In NSABP trials, 14 and 20 patients received lumpectomy plus axillary node dissection or MRM as a surgical procedure. All lumpectomy treated patients were required per protocol to receive standard breast irradiation (without regional nodal irradiation). Chest wall irradiation after mastectomy was not allowed per protocol. Of the 505 patients in both trials that underwent mastectomy, the 10-year Kaplan-Meier LRR, categorised by 21 Gene Assay Recurrence Score (RS) were $2.3 \%, 4.7 \%$ and $16.8 \%$ for low, intermediate and high groups, respectively (log-rank test, $p \geq 0.001$ ) [30].

More recent prospective trial data have suggested certain subsets of pN0 patients may benefit from PMRT, in fact randomised single trial published by Wan showed that patients who receive PMRT had higher DFS that patients who were not irradiated. The main problem with this study is that it did not evaluate LRR, making the data interpretation difficult [48].

\section{Radiation therapy in pathologic stage T3N0 patients}

Several randomised trials and retrospectives studies have addressed this issue in T3N0 patients. The Danish Breast Cancer Collaborative Group trials [49] evaluated the role of PMRT in high-risk patients. Of all randomised patients, 135 premenopausal and 132 postmenopausal women were N0. Postmastectomy radiation decreased locoregional failure (LRF) rate from $17 \%$ to $3 \%$ and $23 \%$ to $6 \%$ in premenopausal and postmenopausal patients, respectively ( $p$ values were not reported) (Table 1). A posterior analysis showed a smaller number of distant metastasis in patients who received PMRT [49]. Of notice, in these studies, axillary lymph node dissection was considered suboptimal with a mean number of 7 lymph nodes removed. Thus, it is possible that some of the reported T3N0 patients could have presented lymph node metastasis if additional axillary lymph nodes had been removed. Since then, several retrospective and population based studies have intended to provide recommendations, but there is no solid prospective data to support PMRT as a standard for N0 tumours larger than 5 $\mathrm{cm}$. Helinto et al [50] in 38 patients reported significant differences of LRR rates between both groups, favouring PMRT. 


\begin{tabular}{|c|c|c|c|c|c|}
\hline 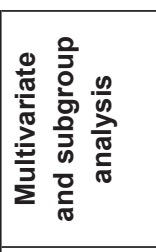 & 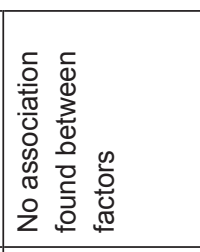 & 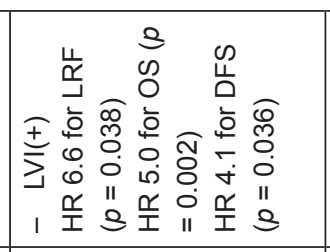 & 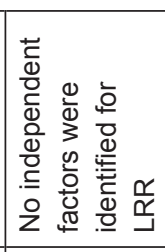 & 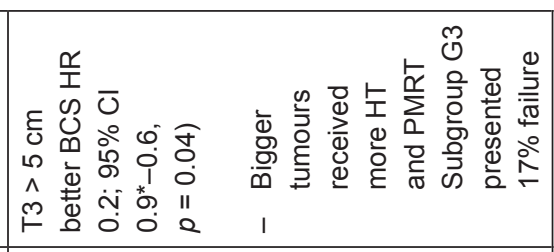 & 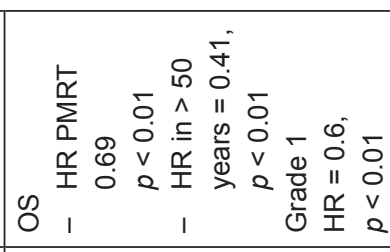 \\
\hline & 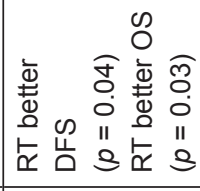 & 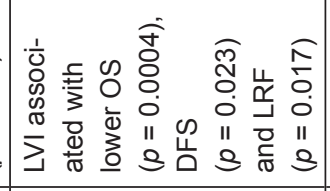 & & & \\
\hline 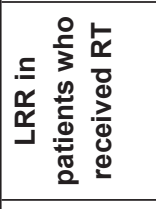 & 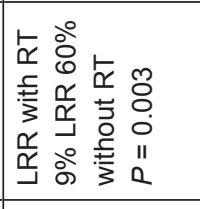 & & & 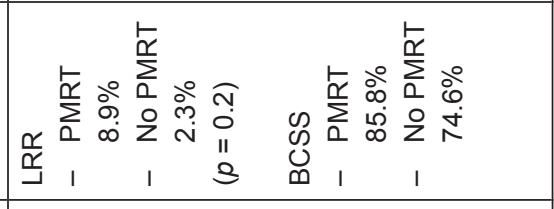 & $\begin{array}{l}\frac{\pi}{10} \\
\frac{\pi}{0} \\
0 \\
\end{array}$ \\
\hline 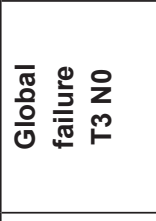 & 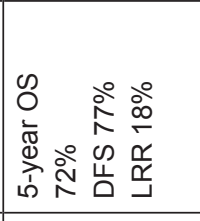 & 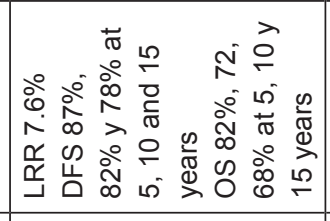 & 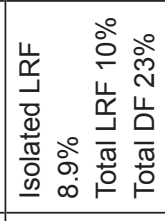 & 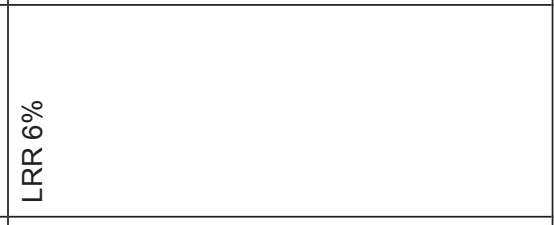 & 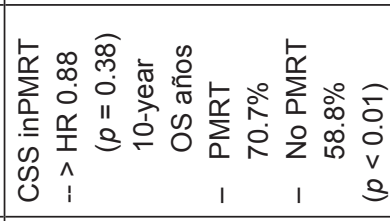 \\
\hline 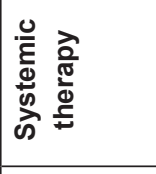 & 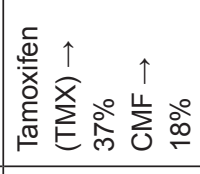 & 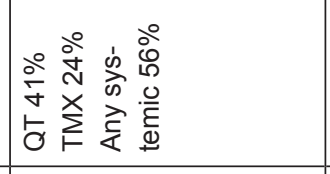 & 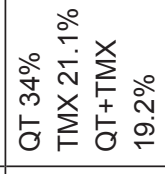 & 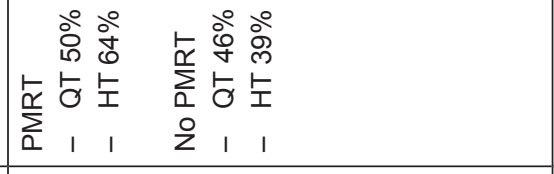 & \begin{tabular}{|l}
$\frac{\pi}{0}$ \\
$\frac{\pi}{0}$ \\
0 \\
2 \\
\end{tabular} \\
\hline 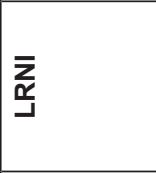 & 总 & z & z & 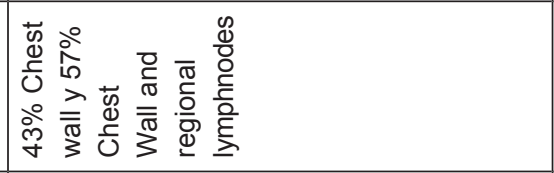 & $\begin{array}{l}\frac{\pi}{0} \\
\frac{\pi}{0} \\
0 \\
z\end{array}$ \\
\hline$\frac{\underline{\alpha}}{\sum_{a}}$ & 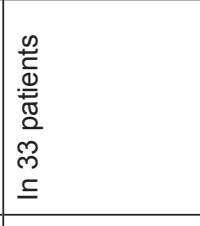 & $z$ & z & 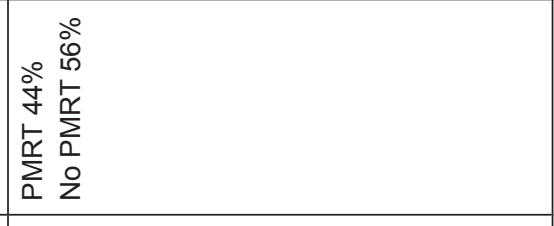 & 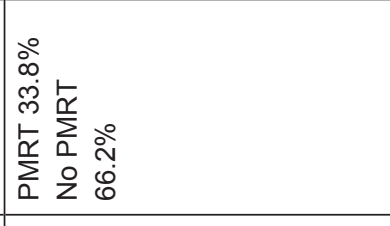 \\
\hline 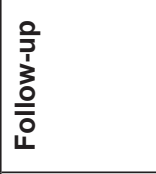 & 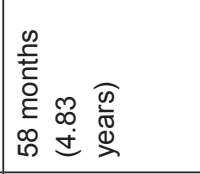 & 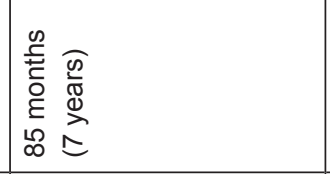 & & 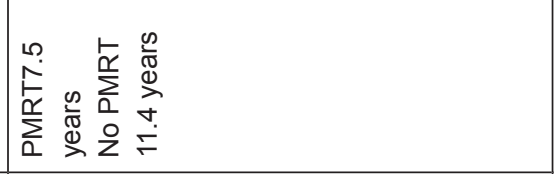 & 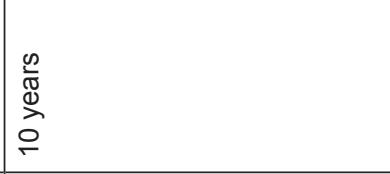 \\
\hline 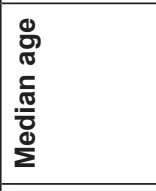 & 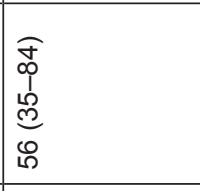 & in & 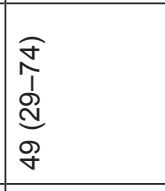 & & $\begin{array}{l}\frac{\pi}{0} \\
\frac{\pi}{8} \\
0 \\
z \\
\end{array}$ \\
\hline 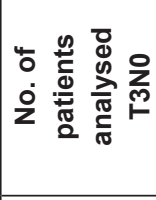 & $\infty$ & R & $\frac{m}{m}$ & 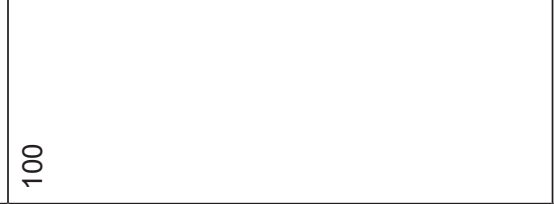 & 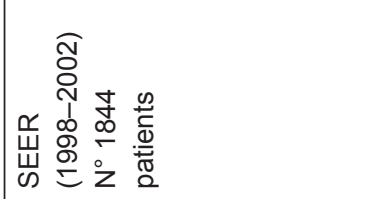 \\
\hline 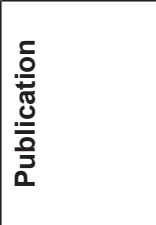 & 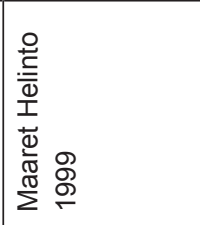 & 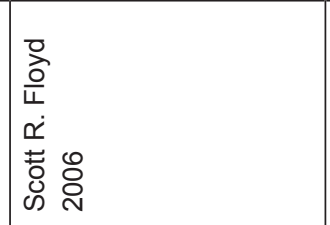 & 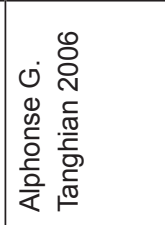 & 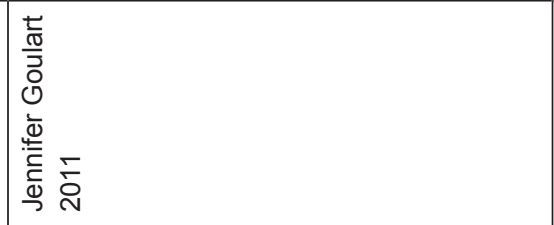 & 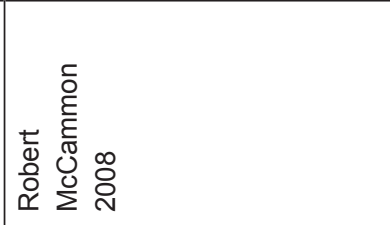 \\
\hline
\end{tabular}




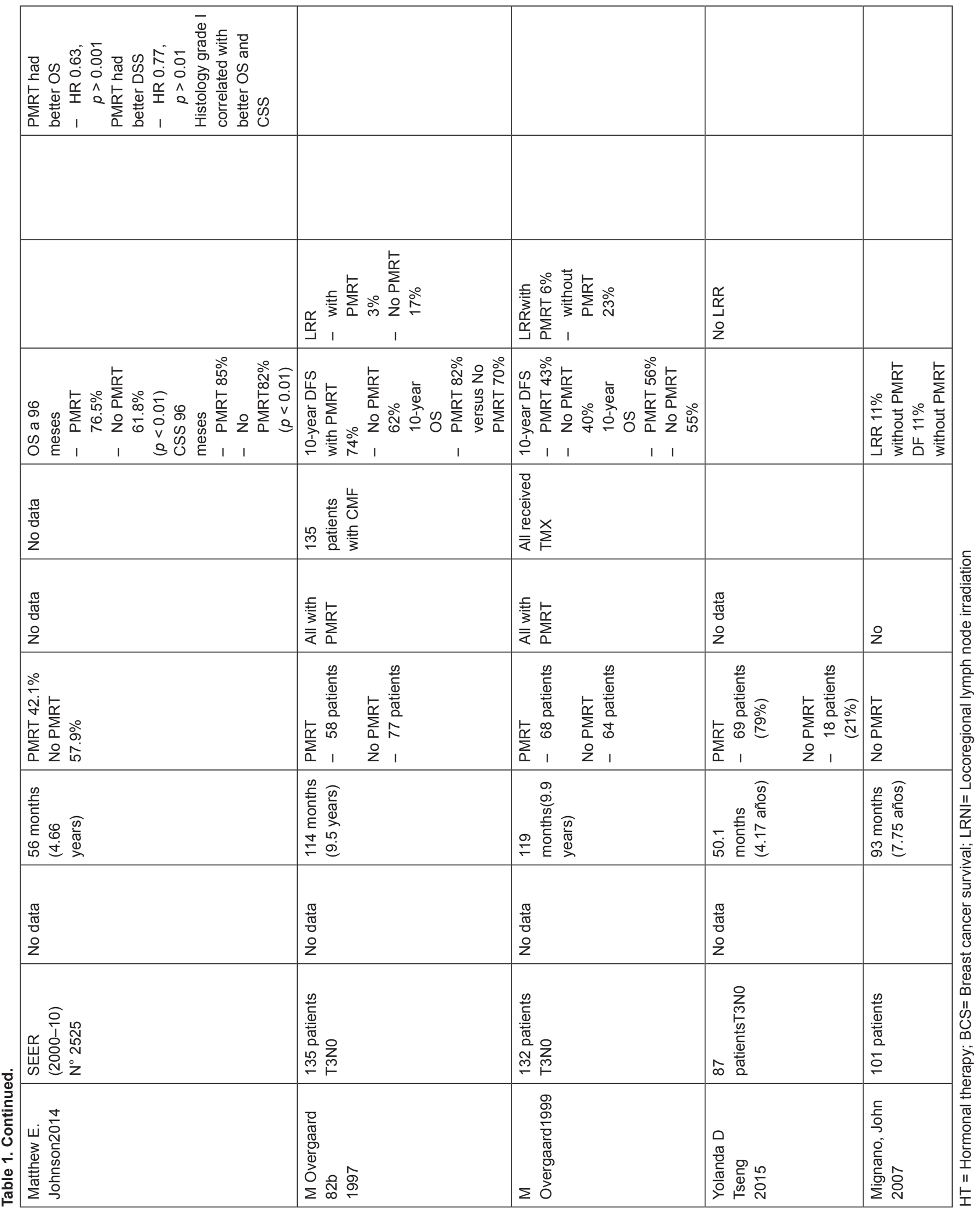


Table 2. T1-T2 N0 PMRT.

\begin{tabular}{|c|c|c|c|c|c|c|c|}
\hline Publication & No. of patients & $\begin{array}{l}\text { Included } \\
\text { population }\end{array}$ & Follow-up & PMRT & $\begin{array}{l}\text { Adjuvant } \\
\text { treatment }\end{array}$ & $\begin{array}{l}\text { LRR global } \\
\text { failure }\end{array}$ & Outcomes \\
\hline \multirow[t]{4}{*}{$\begin{array}{l}2005 \text { Truong } \\
\text { et al }\end{array}$} & 1505 & $\begin{array}{l}\text { T1-2 N0 } \\
\text { with TM }\end{array}$ & 7 years & No & $50.7 \%$ & $7.8 \%$ & Recursive Partitioning Analysis \\
\hline & & & & & & & $\begin{array}{l}10 \text { year Kaplan-Meier LRR G3 versus G1-2 } \\
\rightarrow 12.1 \% \text { versus } 5.5 \%(p<0.0001)\end{array}$ \\
\hline & & & & & & & $\begin{array}{l}10 \text { year Kaplan-Meier } L R R \text { G3+LVI versus } \\
\text { G3 without } L V I \rightarrow 21.2 \% \text { versus } 9 \% \\
(p=0.0008)\end{array}$ \\
\hline & & & & & & & $\begin{array}{l}10 \text { year Kaplan-Meier } \mathrm{LRR} \text { for } \mathrm{G} 3+\mathrm{T} 2+\mathrm{LVI} \\
(-) \text { without systemic treatment versus } \\
\mathrm{G} 3+\mathrm{T} 2+\mathrm{LVI}(-) \text { with systemic therapy } \rightarrow \\
23.2 \% \text { versus } 9.2 \%(p<0.001)\end{array}$ \\
\hline \multirow[t]{8}{*}{$\begin{array}{l}\text { Yildrin } \\
2007\end{array}$} & 502 & $\begin{array}{l}\text { T1-2 N0 } \\
\text { with TM }\end{array}$ & $\begin{array}{l}77 \text { months } \\
\text { (6.4 years) }\end{array}$ & No & $56.2 \%$ & $2.8 \%$ & \\
\hline & & & & & & & $\begin{array}{l}\text { MV analysis in } \leq 40 \text { years }+>2 \mathrm{~cm} \rightarrow \text { LRR } \\
\text { HR } 5.4(p=0.05)\end{array}$ \\
\hline & & & & & & & $\begin{array}{l}\text { MV analysis in } \leq 40 \text { years }+ \text { LVI } \rightarrow \text { LRR HR } \\
9.0(p=0.004)\end{array}$ \\
\hline & & & & & & & $\begin{array}{l}\text { MV analysis in }>40 \text { years }+>3 \mathrm{~cm} \rightarrow \text { LRR } \\
\text { HR } 8.6(p=0.05)\end{array}$ \\
\hline & & & & & & & $\begin{array}{l}\text { MV analysis in }>40 \text { years }+\mathrm{G} 3 \rightarrow \text { LRR HR } \\
7.0(p=0.05)\end{array}$ \\
\hline & & & & & & & $\begin{array}{l}\text { MV analysis in }>40 \text { years }+ \text { LVI } \rightarrow \text { LRR HR } \\
18(p=0.007)\end{array}$ \\
\hline & & & & & & & $\begin{array}{l}\text { Estimated } 10 \text { year LRFS rates: } 98 \% \text { in } \\
\leq 40 \text { years old with } 0-2 \text { RFs (low risk) ver- } \\
\text { sus } 44 \% \text { with }>2 \text { RF (high risk) }(p<0.0001)\end{array}$ \\
\hline & & & & & & & $\begin{array}{l}\text { Estimated } 10 \text { year LRFS rates: } 99 \% \text { in }<3 \\
\text { RF (low risk) in }>40 \text { years old versus } 57 \% \text { in } \\
\text { patients }>3 \text { RFs (high risk) }(p<0.0001)\end{array}$ \\
\hline \multirow[t]{4}{*}{$\begin{array}{l}\text { Trovo } \\
2012\end{array}$} & $159 \rightarrow 54 \%$ No & $\begin{array}{l}\text { T1-2 N0-1 } \\
\text { with TM }\end{array}$ & $\begin{array}{l}75 \text { months } \\
(6.25 \\
\text { years })\end{array}$ & PMRT in N1 & $95 \%$ & $11 \%$ & $\begin{array}{l}5 \text { year Kaplan-Meier LRR in Patients LVI } \\
(+) \text { versus LVI }(-) \rightarrow 19.1 \% \text { versus } 3.2 \% \\
(p=0.002)\end{array}$ \\
\hline & & & & & & & $\begin{array}{l}\text { Kaplan-Meier LRR in Premenopausal } \\
\text { versus postmenopausal status } \rightarrow 13.4 \% \\
\text { versus } 4.8 \%(p=0.004)\end{array}$ \\
\hline & & & & & & & $\begin{array}{l}\text { Kaplan-Meier LRR inER }(-) \text { versus ER }(+) \\
\rightarrow 25.8 \% \text { versus } 4.7 \%(p=0.002)\end{array}$ \\
\hline & & & & & & & $\begin{array}{l}\text { Kaplan-Meier LRR in G3 versus G1-2 } \rightarrow \\
16.4 \% \text { versus } 1.4 \%(p=0.002) \\
\text { Kaplan-Meier LRR in patients with non or } \\
\text { one, two, three or four LRR factors } \rightarrow 1.2 \% \text {, } \\
10.3 \%, 24.1 \% \text { and } 75 \%(p<0.001)\end{array}$ \\
\hline $\begin{array}{l}\text { Rita } \\
\text { Abi-Raad } \\
2011\end{array}$ & 1136 & $\begin{array}{l}\text { T1-2N0 } \\
\text { with TM }\end{array}$ & 9 years & No & $61.6 \%$ & $5.2 \%$ & $\begin{array}{l}\text { LRR MV analysis in patients with Systemic } \\
\text { treatment } \rightarrow \text { HR } 0.5(p=0.036)\end{array}$ \\
\hline
\end{tabular}


Table 2. Continued.

\begin{tabular}{|c|c|c|c|c|c|c|c|}
\hline & & & & & & & $\begin{array}{l}\text { LRR MV analysis in patients with margins } \\
<2 \mathrm{~mm} \rightarrow \mathrm{HR} 3.3(p=0.001)\end{array}$ \\
\hline & & & & & & & $\begin{array}{l}\text { LRR MV analysis in patients with Size } \\
\geq 2 \mathrm{~cm} \rightarrow \operatorname{HR} 2.0(p=0.011)\end{array}$ \\
\hline & & & & & & & $\begin{array}{l}\text { LRR MV analysis in patients with }>50 \text { years } \\
\text { old } \rightarrow \text { HR } 0.5(p=0.018)\end{array}$ \\
\hline & & & & & & & $\begin{array}{l}\text { LRR MV analysis in patients with ILV }(+) \rightarrow \\
\text { HR } 2.7(p=0.002)\end{array}$ \\
\hline & & & & & & & $\begin{array}{l}10 \text { year LRR Kaplan-Meier without RFs } 2 \% \text {; } \\
\text { LRR with } 1 \text { RF } 3.3 \% \text {; LRR with } 2 \text { RF } 5.8 \% \text {; } \\
\geq 3 \text { RF, } 19.7 \%(p<0.0001)\end{array}$ \\
\hline \multirow[t]{5}{*}{$\begin{array}{l}\text { Xing Xing } \\
\text { Chen } 2013\end{array}$} & $\begin{array}{l}390 \rightarrow 307 \mathrm{N0} \\
(78.7 \%)\end{array}$ & $\begin{array}{l}\text { TNBC T1-2 } \\
\text { N0-1 with } \\
\text { TM }\end{array}$ & $\begin{array}{l}60.5 \\
\text { months } \\
\text { (5 years) }\end{array}$ & No & $86 \%$ & $7.9 \%$ global & $\begin{array}{l}\text { RR MV analysis in patients }<50 \text { years } \\
\text { versus } \geq 50 \text { years } \rightarrow \text { HR } 4.82(p=0.015)\end{array}$ \\
\hline & & & & & & & $\begin{array}{l}\text { LRR MV analysis in patients with } \mathrm{N}(+) 3 \\
\text { versus } 0-2 \rightarrow \mathrm{HR} 8.76(p=0.03)\end{array}$ \\
\hline & & & & & & & $\begin{array}{l}\text { LRR MV analysis in patients with LVI }(+) \\
\text { versus LVI }(-) \rightarrow \text { HR } 26.05(p=0.000)\end{array}$ \\
\hline & & & & & & & $\begin{array}{l}\text { LRR MV analysis in patients with G3 tumour } \\
\text { versus } \mathrm{G} 1-2 \rightarrow \mathrm{HR} 2.87(p=0.039)\end{array}$ \\
\hline & & & & & & & $\begin{array}{l}5 \text { year KM LRR with } 0-1 \text { RF was } 4.2 \% \text {; with } \\
2 \text { RF } 25.2 \% \text {; with }>3 \text { RF } 81 \%(p<0.001)\end{array}$ \\
\hline \multirow[t]{3}{*}{$\begin{array}{l}\text { Ranjna } \\
\text { Sharma } \\
2010\end{array}$} & $1019 \rightarrow 753$ No & $\begin{array}{l}\text { T1-2N0-1 } \\
\text { with TM }\end{array}$ & 7.47 years & No & $76.9 \%$ & $2.3 \%$ & $\begin{array}{l}10 \text { year Kaplan-Meier LRR in N0 patients } \\
\leq 40 \text { years versus }>40 \text { years } \rightarrow 10.5 \% \\
\text { versus } 1 \%(p<0.0001)\end{array}$ \\
\hline & & & & & & & $\begin{array}{l}\text { LRR MV analisys in N0 patients } \leq 40 \text { years } \\
\rightarrow \text { HR } 2.14(p=0.004)\end{array}$ \\
\hline & & & & & & & $\begin{array}{l}10 \text { year Kaplan-Meier LRR in T1N0 and } \\
\text { T2N0 in } \leq 40 \text { years old patients was } 9.3 \% \\
\text { and } 18.6 \% \text { ( } p \text { not reported) }\end{array}$ \\
\hline \multirow[t]{3}{*}{$\begin{array}{l}\text { Troung } \\
2014\end{array}$} & 1994 pacientes & $\begin{array}{l}\text { T1-2 N0 } \\
\text { with TM }\end{array}$ & 4.3 years & No & $80.5 \%$ & & $\begin{array}{l}\text { LRR MV analisys Lobular histology } \rightarrow \mathrm{HR} \\
3.48(p=0.003)\end{array}$ \\
\hline & & & & & & & $\begin{array}{l}\text { LRR MV analisys Close or positive } \\
\text { margins } \rightarrow \mathrm{HR} 3.42(p=0.009)\end{array}$ \\
\hline & & & & & & & $\begin{array}{l}\text { LRR MV analisys Tumour size }>2 \mathrm{~cm} \rightarrow \\
\text { HR } 2.57(p=0.012)\end{array}$ \\
\hline \multirow[t]{2}{*}{$\begin{array}{l}\text { Jessica Selz } \\
2012\end{array}$} & 699 patients & $\begin{array}{l}\text { T1-2 N0 } \\
\text { with TM }\end{array}$ & $\begin{array}{l}56 \text { months } \\
\text { (4.6 years) }\end{array}$ & $\begin{array}{l}\text { - } 191 \text { PMRT } \\
\text { with RLI } \\
-\quad 508 \text { with- } \\
\text { out PMRT }\end{array}$ & $\begin{array}{l}\text { HT } 65.5 \% \\
\text { CT } 33.5 \% \\
\text { Trastuzumab } \\
3.7 \%\end{array}$ & $\begin{array}{l}\text { Freedom } \\
\text { from LRR: } \\
97 \% \\
\text { patients }\end{array}$ & $\begin{array}{l}\text { - LRR MV analisys in patients with with } \\
\text { Ki67 }>20 \% \rightarrow \text { HR } 4.18(p<0.0215)\end{array}$ \\
\hline & & & & & & & $\begin{array}{l}5 \text { year LRRFS in PMRT versus no PMRT } \\
\text { patients } \rightarrow 97.7 \% \text { versus } 96.8 \%(p=0.663)\end{array}$ \\
\hline
\end{tabular}


Table 2. Continued.

\begin{tabular}{|c|c|c|c|c|c|c|c|}
\hline \multirow[t]{2}{*}{$\begin{array}{l}\text { Bassam S. } \\
\text { Abdulkarim } \\
2011\end{array}$} & $\begin{array}{l}768 \text { TNBC } \\
-\quad \text { BCT+RT in } \\
\text { T1-2 N0 } \rightarrow \\
233 \\
-\quad \text { T1-2N0 } \\
\quad \text { MT with no } \\
\text { PMRT } \rightarrow \\
235\end{array}$ & $\begin{array}{l}\text { T1-2N0 } \\
\text { with TM }\end{array}$ & 7.2 years & No & $\begin{array}{l}69.9 \% \text { in } \\
\text { those with } \\
\text { TM }\end{array}$ & LRR 10\% & $\begin{array}{l}\text { LRR MV analisys for MRM without RT } \\
\text { versus BCT+RT in T1-2 N0 } \rightarrow \text { HR } 3.44 \\
(p<0.001)\end{array}$ \\
\hline & & & & & & & $\begin{array}{l}\text { LRR MV analisys for MRM with RT versus } \\
\text { BCT+RT in T1-2 N0 } \rightarrow 0.72(p=0.34)\end{array}$ \\
\hline \multirow{3}{*}{$\begin{array}{l}\text { Joseph } \\
\text { Hastings } \\
2014\end{array}$} & 1259 patients & $\begin{array}{l}\text { TNBC T1 } \\
\text { N0 with TM }\end{array}$ & 8.5 years & No & QT $24.2 \%$ & $\begin{array}{l}10 \text { year } \\
\text { LRR } 3.2 \%\end{array}$ & $\begin{array}{l}\text { LRR MV analysis in patients with margins } \\
\leq 3 \mathrm{~mm} \rightarrow \mathrm{HR} 2.97(p=0.02)\end{array}$ \\
\hline & & & & & & & $\begin{array}{l}\text { LRR MV analysis in patients in patien with } \\
\text { G3 versus } \mathrm{G} 1-2 \text { tumours } \rightarrow \text { HR } 3.97 \\
(p=0.0002)\end{array}$ \\
\hline & & & & & & & $\begin{array}{l}10 \text { year Kaplan-Meier LRR in Patients with } \\
\text { one of these RFs versus two RFs } \rightarrow 2.7 \% \\
\text { versus } 25 \%(p<0.0001)\end{array}$ \\
\hline
\end{tabular}

$\mathrm{G}=$ histological grade; $\mathrm{BCT}=$ breast conservative treatment; $\mathrm{NS}=$ not significant $\mathrm{RLI}=$ regional lymph node irradiation

Floyd et al [51] reported retrospectively a series of 70 T3N0 breast cancer patients who received radical mastectomy and systemic therapy without RT between 1981 and 2002. At a median follow-up of 85 months, 5-year survival and LRF rates were $83 \%$ and $7.6 \%$, respectively. Of note, LVI was associated with higher LRR by log rank test $(P=0.017)$ with actuarial local failure rate of $21 \%$ for patients with LVI versus $4 \%$ for patients without LVI (Table 1). In the multivariate analysis, LVI status was significantly associated with lower locoregional free survival (LRFS) $(p=0.038, \mathrm{HR}=6.6)$, OS ( $p=0.002$, HR 5.0) and DFS ( $p=0.036$, HR 4.1), respectively. Taghian et al [52] evaluated 313 T3N0 mastectomised patients, without PMRT, treated in NSABP node negative trials (NSABP B-13, B-14, B-19, B-20 and B-23). Overall, 10-year cumulative incidence of isolated LRR was $7.1 \%$. LRF with DF was $10.0 \%$, while DF alone was $23.6 \%$. Multivariate analysis did not identify significant prognostic factors for LRR. The authors concluded that PMRT should not be routinely used for these patients. A criticism of this study was that it did not evaluate all negative features for LRR.

Goulart et al [53] investigated 100 T3N0 breast cancer patients; 44 (44\%) patients received adjuvant PMRT. At a median follow-up of 10 years, there was no difference in cumulative LRR rates between both groups; as depicted by $8.9 \%$ and $2.3 \%$ LRR rates in the no-PMRT and PMRT groups, respectively $(P=0.2)$. To consider, better BCSS rates were reported for patients with tumours $>5 \mathrm{~cm}$ and margin positives cases, compared to the smaller tumours. This was attributed to the fact that a greater number of patients with larger tumours received PMRT when compared to smaller tumours (18\% versus $54 \% ; p=0.001)$. In addition, a greater number of patients with larger tumours received hormonotherapy $(57 \%$ versus $32 \% ; p=0.03)$.

From a single institution database, Mignano et al [54] retrospectively evaluated 101 T3N0 breast cancer patients treated with mastectomy without PMRT, enrolled from 1974 to 1994. At a median follow-up of 93 months, LRR was $11 \%$. There was no significant difference in LRR between tumours smaller or larger than $7 \mathrm{~cm}(p=0.07)$.

Two studies from the Surveillance, Epidemiology and End Results (SEER) program database found mixed results. McCammon et al [55] performed a retrospective population-based analysis which included 1,844 women undergoing mastectomy and axillary staging from 1988 to 2002 , considering a maximum follow-up of 180 months. Only a $33.8 \%$ of the sample received PMRT. The authors reported no significant differences in CSS when compared PMRT versus no PMRT. However, patients who received PMRT presented an OS of $70.7 \%$, whereas patients who did not receive PMRT had OS rates of $58.4 \%$ with a median follow-up of 10 years $(\mathrm{HR}, 0.69 ; 95 \% \mathrm{Cl} 0.55-0.85, p<0.01)$. 
The other SEER database study [56] was performed in 2,525 women who underwent MRM between 2000 and 2010; 42\% of the cohort received PMRT. At a median follow-up of 56 months, PMRT was associated with both OS and CSS with an HR of $0.63(p<0.001)$ and $0.77(p=0.045)$, respectively. These studies were not designed to evaluate LRR, but apparently PMRT would have had a protective role in terms of OS and CSS.

\section{Discussion}

Several studies have attempted to describe correlations between molecular subtypes and outcomes for breast cancer disease control and survival. Traditionally, it has been described that Her2neu positive disease and TNBC have an increased risk of LRR compared to Luminal A subtype. In their study of 2,985 patients, Voduc et al [25], Kyndi et al [26], Chen et al [36] and Albert et al [57] found significant differences in LRR rates based on molecular subtypes. The investigators reported LRFS and regional relapse-free survival of $92 \%$ and $96 \%$ for Luminal A subtype; $86 \%$ and $88 \%$ in Luminal B; $80 \%$ and $88 \%$ in Her2 (+) Luminal; $83 \%$ and $88 \%$ in Her2 (+) no luminal, and finally, $80 \%$ and $88 \%$ in TNBC, respectively. Tumour size and histological grade were found to be independent prognostic factors for this outcome.

Similarly, a series from MD Anderson Cancer Centre [58] described LRR rates of $28 \%$ in TNBC. Independent RFs for LRR in mastectomised patients were tumour size, positive margin, grade, presence of LVI and the lack of anthracycline-based QT.

Zumsteg et al [59] in a single institutional study evaluated 642 patients with T1-2 N0 disease. TNBC patients who were treated with TM presented an LRR rate of $5.4 \%$ versus $4.2 \%$ that went to conservative therapy. This suggests study a low recurrence risk instead of aggressive biology. Despite TNBC $[29,36,39,57]$ are associated with higher LRR and worse outcomes, molecular biology should not be the only factor to take into consideration because by itself it might be not important enough.

LVI has also been associated with BCSS and LRR [60-62]. Lee et al [61] analysed 2,760 node negative breast cancer patients in order to evaluate BCSS. They observed that LVI was associated with increasing tumour size $(p<0.0001)$, higher histological grade $(p<0.0001)$, less favourable Nottingham Prognostic Index group $(p<0.0001)$ and younger age $(p<0.0001)$. Moreover, LVI was a significant prognostic factor in the multivariate analysis for BCSS in patients with and without adjuvant therapy. In the context of LRR, Truong et al [40] observed on 763 T1-2N0 patients, at a median follow up of 7 years, that LVI was an independent prognostic factor for LRR (RR 2.32; 95\% Cl 1.26-4.27, $p<0.007$ ). In the same way, Boutrus et al [62] studied 1.275 N0 women (692 premenopausal and 583 postmenopausal) who underwent mastectomy followed by systemic therapy. They identified LVI $(p=0.23)$ and tumour size $>2 \mathrm{~cm}(p=0.027)$ as RFs for LRR in premenopausal women, while LVI was the only significant factor associated with loco-regional failure in postmenopausal women.

Wallgren et al [63] reviewed the records of 1.257 T1-3N0-1 patients treated with BCT between 1980 and 2003. The aim of the study was to evaluate LVI as a predictor of nodal failure. At median follow-up of 8 years (ranged, 0.1-21 years), LVI was present in 17\% of patients. In the multivariate analysis, tumour size, high grade and local failure were significant predictors of regional nodal failure $(p=0.049, p=0.013$, $p=0.0001$, respectively), whereas LVI did not show a significant relationship with regional nodal failure. The presence of LVI in T2 and T3 population did not increase the risk of regional nodal failure $(p=0.15)$. These results suggest that LVI should not be used as a sole indicator for regional failure. This is reaffirmed by a recent study in which it is shown that LVI is not the only factor to be taken into consideration when categorising a patient as having a high risk of failure. Although this study did not exclusively analyse patients, N0 is applicable to this group and to all patients with breast cancer [64].

Tumour size is another important prognostic factor associated with $\operatorname{LRR}[13,28,34,53]$. As tumour size increases, so does the risk of nodal compromise, and chance to develop distant metastases and mortality as well. In 1989, Carter et al [65] postulated that tumour diameter and nodal status were independent but additive in-patient's prognosis. They established a linear relationship between size and nodal compromise. Similarly, high-grade tumours have been correlated with increased risk of recurrence and worse prognosis [5, 31].

Ki67, a cell proliferation marker, has been described as a prognostic factor for LRR after neoadjuvant QT [66]. Synnestvedt et al [67] reported an inverse correlation between high Ki67 levels and DFS, DDFS and CSS.

Similarly, Kilickap et al [68] correlated high Ki67 levels with ER (-), HER2 new positive and higher tumour grade. The investigators also reported that high Ki67 levels were correlated with more failures and decreased survival. Nevertheless, up to date, Ki67 has not been validated as a marker able to predict recurrence and thus should be used in association with other factors based on clinical judgment [68]. 
Another important variable is pathology quality assurance. The BIG 2.04 SUPREMO phase III trial evaluated pathologic inclusion criteria entry for the trial RT postmastectomy for intermediate-risk breast cancer and suggests after central review that $19 \%$ of node negative patients were ineligible for trial. These data raise questions about whether clinical trials need to be powered to accommodate significant minorities of patients actually being ineligible, or should they reflect practice in the real world [69]?

It is true that there are reports in the specific group of young patients, that the main risk is distance failure, rather than LRR, it should be considered that breast cancer in patients $<40$ years old, age is a RF for treatment failure (Table 1), independent of molecular subtype [70]. Azim et al analysed 1.360 patients with pT1-2 N0 tumours treated with BCT and found a local recurrence hazard ratio in women $<45$ years old of 4.09 compared to women $>65$ years old.

Younger patients tend to harbour more aggressive disease with higher rates of local failure and decreased survival. Therefore, we suggest that a multidisciplinary team should perform their evaluation and management.

\section{Conclusion}

The decision to carry out PMRT to reduce the risk of LRF must be personalised. In this context, the use of PMRT in breast cancer patients with tumour size $\geq 5 \mathrm{~cm}$ must be recommended in the presence of one or more RFs such as young age (<40 years or premenopausal status), no use of systemic treatment, large tumour size, margin status, LVI and molecular subtype due to the high risk of LRR. There is no evidence for PMRT in absence of these factors.

In the group of patients with T1-2N0 disease who have undergone mastectomy, we conclude that the decision of PMRT should be personalised and discussed, based on the knowledge of the disease, the clinical-pathological factors that affect it and the molecular factors that might influence the results, always in a multidisciplinary team with all those involved in the treatment of the patient. Also this treatment should be discussed with the patient, exposing the risks and benefits of the LRR and treatment. Despite the limitations of the studies analysed in this review and the fact that there is no prospective evidence to support this treatment, we considered the possibility of PMRT in patients with high risk of LRR.

\section{Conflicts of interest}

None.

\section{Funding}

The authors have no funding to report

\section{Abbreviations}

BCSS Breast cancer specific survival

BCT Breast conservative treatment

CSS Cancer specific survival

DF Distant failure

DFS Disease free survival 


$\begin{array}{ll}\text { EBCTCG } & \text { Early Breast Cancer Trialists' Collabora } \\ \text { ER } & \text { Estrogen receptor } \\ \text { HT } & \text { Hormonal Therapy } \\ \text { LRF } & \text { Locoregional failure } \\ \text { LRFS } & \text { Locoregional free survival } \\ \text { LRNI } & \text { Locoregional lymph node irradiation } \\ \text { LRR } & \text { Locoregional recurrence } \\ \text { LVI } & \text { Lymphovascular invasion } \\ \text { MRM } & \text { Modified radical mastectomy } \\ \text { N(-) } & \text { Node negative } \\ \text { N(+) } & \text { Node positive } \\ \text { OS } & \text { Overall survival } \\ \text { PMRT } & \text { Postmastectomy radiotherapy } \\ \text { QT } & \text { Chemotherapy } \\ \text { RT } & \text { Radiotherapy } \\ \text { TM } & \text { Total mastectomy } \\ \text { TMX } & \text { Tamoxifen } \\ \text { TNBC } & \text { Triple negative breast cancer } \\ \end{array}$

\section{References}

1. [http://www.cancer.org/content/dam/cancer-org/research/cancer-facts-and-statistics/annual-cancer-facts-and-figures/2017/cancerfacts-and-figures-2017.pdf]

2. Howlader N, NooneAM, and Krapcho M, et al (2016) SEER Cancer Statistics Review, 1975-2013 (Bethesda, MD: National Cancer Institute) [http://seer.cancer.gov/csr/1975 2013/, based on November 2015 SEER data submission, posted to the SEER web site]

3. Narod SA, Iqbal J, and Miller AB (2015) Why have breast cancer mortality rates declined? J Cancer Policy 5 8-17 https://doi. org/10.1016/j.jcpo.2015.03.002

4. Overgaard M, Jensen MB, and Overgaard J, et al (1999) Postoperative radiotherapy in high-risk postmenopausal breastcancer patients given adjuvant tamoxifen: Danish Breast Cancer Cooperative Group DBCG 82c randomised trial Lancet 353 1641-1648 https://doi.org/10.1016/S0140-6736(98)09201-0 PMID: 10335782

5. Overgaard M, Hansen PS, and Overgaard J, et al (1997) Postoperative radiotherapy in high-risk premenopausal women with breast cancer who receive adjuvant chemotherapy. Danish Breast Cancer Cooperative Group 82b Trial N Engl J Med 337(14) 949-955 https://doi.org/10.1056/NEJM199710023371401

6. Ragaz J, Olivotto IA, and Spinelli JJ, et al Locoregional radiation therapy in patients with high-risk breast cancer receiving adjuvant chemotherapy: 20-year results of the British Columbia randomized trial J Natl Cancer Inst 97 116-126 PMID: 15657341 
7. EBCTCG, McGale P, and Taylor C, et al (2014) Effect of radiotherapy after mastectomy and axillary surgery on 10-year recurrence and 20-year breast cancer mortality: meta-analysis of individual patient data for 8135 women in 22 randomised trials Lancet 383 2127-2135 https://doi.org/10.1016/S0140-6736(14)60488-8 PMID: 24656685 PMCID: 5015598

8. Whelan TJ, Pignol JP, and Levine MN, et al (2010) Long-term results of hypofractionated radiation therapy for breast cancer N Engl J Med 362 513-520 https://doi.org/10.1056/NEJMoa0906260 PMID: 20147717

9. Haviland JS, Owen JR, and Dewar JA, et al (2013) The UK Standardisation of Breast Radiotherapy (START) trials of radiotherapy hypofractionation for treatment of early breast cancer: 10-year follow-up results of two randomised controlled trials Lancet Oncol 14 1086-1094 https://doi.org/10.1016/S1470-2045(13)70386-3 PMID: 24055415

10. Friedel G, Kuipers $T$, and Dippon J, et al (2008) Full-thickness resection with myocutaneous flap reconstruction for locally recurrent breast cancer Ann Thorac Surg 85 1894-1900 https://doi.org/10.1016/j.athoracsur.2008.02.012 PMID: 18498790

11. Skinner HD, Strom EA, and Motwani SB, et al (2013) Radiation dose escalation for loco-regional recurrence of breast cancer after mastectomy Radiat Oncol 813 https://doi.org/10.1186/1748-717X-8-13 PMID: 23311297 PMCID: 3552737

12. Ballo MT, Strom EA, and Prost $\mathrm{H}$ et al (1999) Local-regional control of recurrent breast carcinoma after mastectomy: does hyperfractionated accelerated radiotherapy improve local control? Int J Radiat Oncol Biol Phys 44 105-112 https://doi.org/10.1016/ S0360-3016(98)00545-8 PMID: 10219802

13. Fisher B, Anderson S, and Fisher ER et al (1991) Significance of ipsilateral breast tumor recurrence after lumpectomy Lancet 338 327-331 https://doi.org/10.1016/0140-6736(91)90475-5 PMID: 1677695

14. Shenouda MN, Sadek BT, and Goldberg SI, et al (2014) Clinical outcome of isolated locoregional recurrence in patients with breast cancer according to their primary local treatment Clin Breast Cancer 14 198-204 https://doi.org/10.1016/j.clbc.2013.12.007 PMID: 24485702

15. Rauschecker H, Clarke M, and Gatzemeier W, et al (2001) Systemic therapy for treating locoregional recurrence in women with breast cancer Cochrane Database Syst Rev CD002195 PMID: 11687148

16. Aebi S, Gelber S, and Anderson SJ, et al (2014) Chemotherapy for isolated locoregional recurrence of breast cancer (CALOR): a randomised trial Lancet Oncol 15 156-163 https://doi.org/10.1016/S1470-2045(13)70589-8 PMID: 24439313 PMCID: 3982874

17. Wapnir IL, Gelber S, and Anderson SJ, et al (2017) Poor prognosis after second locoregional recurrences in the CALOR trial Ann Surg Oncol 24(2) 398-406 https://doi.org/10.1245/s10434-016-5571-y PMCID: $\underline{5215961}$

18. Witteveen A, Kwast ABG, and Sonke GS, et al (2015) Survival after locoregional recurrence or second primary breast cancer: impact of the disease-free interval PLoS One 10(4) e0120832 https://doi.org/10.1371/journal.pone.0120832 PMID: 25861031 PMCID: 4393268

19. Wang W (2013) Radiotherapy in the management of early breast cancer J Med Radiat Sci 60(1) 40-46 https://doi.org/10.1002/ imrs.1 PMID: 26229606 PMCID: $\underline{4175791}$

20. Darby SC, Ewertz M, and McGale P, et al (2013) Risk of ischemic heart disease in women after radiotherapy for breast cancer N Engl J Med 368 987-998 https://doi.org/10.1056/NEJMoa1209825 PMID: 23484825

21. Blom Goldman U, Wennberg B, and Svane G, et al (2010) Reduction of radiation pneumonitis by V20-constraints in breast cancer Radiat Oncol 599 https://doi.org/10.1186/1748-717X-5-99 PMID: 21034456 PMCID: 2987943

22. Gagliardi G, Constine LS, and Moiseenko V, et al (2010) Radiation dose-volume effects in the heart Int J Radiat Oncol Biol Phys 76(3 Suppl) 77-85 https://doi.org/10.1016/j.jijobp.2009.04.093

23. Perou CM, Sørlie T, and Eisen MB, et al (2000) Molecular portraits of human breast tumours Nature 406 747-752 https://doi. org/10.1038/35021093 PMID: 10963602 
24. Sorlie T, Perou CM, and Tibshirani R, et al (2001) Gene expression patterns of breast carcinomas distinguish tumor subclasses with clinical implications Proc Natl Acad Sci U S A 98 10869-10874 https://doi.org/10.1073/pnas.191367098 PMID: 11553815 PMCID: $\underline{58566}$

25. Voduc KD, Cheang MC, and Tyldesley S, et al (2010) Breast cancer subtypes and the risk of local and regional relapse $J$ Clin Oncol 28 1684-1691 https://doi.org/10.1200/JCO.2009.24.9284 PMID: 20194857

26. Kyndi M, Sørensen FB, and Knudsen H, et al (2008) Estrogen receptor, progesterone receptor, HER-2, and response to postmastectomy radiotherapy in high-risk breast cancer: the Danish Breast Cancer Cooperative Group J Clin Oncol 26 1419-1426 https://doi.org/10.1200/JCO.2007.14.5565 PMID: 18285604

27. Parise CA and Caggiano V (2014) Breast cancer survival defined by the ER/PR/HER2 subtypes and a surrogate classification according to tumor grade and immunohistochemical biomarkers J Cancer Epidemiol 2014469251 https://doi. org/10.1155/2014/469251 PMID: 24955090 PMCID: 4058253

28. Truong PT, Sadek BT, and Lesperance MF, et al (2014) Is biological subtype prognostic of locoregional recurrence risk in women with pT1-2N0 breast cancer treated with mastectomy? Int J Radiat Oncol Biol Phys 88 57-64 https://doi.org/10.1016/j. ijrobp.2013.09.024

29. Abdulkarim BS, Cuartero J, and Hanson J, et al (2011) Increased risk of locoregional recurrence for women with T1-2N0 triplenegative breast cancer treated with modified radical mastectomy without adjuvant radiation therapy compared with breastconserving therapy J Clin Oncol 29 2852-2858 https://doi.org/10.1200/JCO.2010.33.4714 PMID: 21670451 PMCID: $\underline{5073381}$

30. Mamounas EP, Tang G, and FisherB, et al (2010) Association between the 21-gene recurrence score assay and risk of loco regional recurrence in node-negative, estrogen receptor-positive breast cancer: results from NSABP B-14 and NSABP B-20 J Clin Oncol 28(10) 1677-1683 https://doi.org/10.1200/JCO.2009.23.7610 PMID: 20065188 PMCID: 2849763

31. Nottage MK, Kopciuk KA, and Tzontcheva A, et al (2006) Analysis of incidence and prognostic factors for ipsilateral breast tumour recurrence and its impact on disease-specific survival of women with node-negative breast cancer: a prospective cohort study Breast Cancer Res 8 R44 https://doi.org/10.1186/bcr1531 PMID: 16859523 PMCID: 1779479

32. Katz A, Strom EA, and Buchholz TA, et al (2000) Locoregional recurrence patterns after mastectomy and doxorubicin-based chemotherapy: implications for postoperative irradiation J Clin Oncol 18 2817-2827 https://doi.org/10.1200/JC0.2000.18.15.2817 PMID: 10920129

33. Truong PT, Yong CM, and Abnousi F, et al (2005) Lymphovascular invasion is associated with reduced locoregional control and survival in women with node-negative breast cancer treated with mastectomy and systemic therapy J Am Coll Surg 200 912-921 https://doi.org/10.1016/j.jamcollsurg.2005.02.010 PMID: 15922205

34. Abi-Raad R, Boutrus R, and Wang R, et al (2011) Patterns and risk factors of locoregional recurrence in T1-T2 node negative breast cancer patients treated with mastectomy: implications for postmastectomy radiotherapy Int J Radiat Oncol Biol Phys 81 e151-e157 https://doi.org/10.1016/j.jirobp.2011.01.015 PMID: 21420245 PMCID: 3722592

35. Kiess AP, McArthur HL, and Mahoney K, et al (2012) Adjuvant trastuzumab reduces locoregional recurrence in women who receive breast-conservation therapy for lymph node-negative, human epidermal growth factor receptor 2-positive breast cancer Cancer 118 1982-1988 https://doi.org/10.1002/cncr.26484

36. Chen X, Yu X, and Chen J, et al (2013) Analysis in early stage triple-negative breast cancer treated with mastectomy without adjuvant radiotherapy: patterns of failure and prognostic factors Cancer 119 2366-2374 https://doi.org/10.1002/cncr.28085 PMID: 23576181

37. Yildirim $E$ and Berberoglu $U$ (2007) Can a subgroup of node-negative breast carcinoma patients with T1-2 tumor who may benefit from postmastectomy radiotherapy be identified? Int J Radiat Oncol Biol Phys 68 1024-1029 https://doi.org/10.1016/j. ijrobp.2007.01.015 PMID: $\underline{17398017}$ 
38. Jwa E, Shin KH, and Lim HW, et al (2015) Identification of risk factors for locoregional recurrence in breast cancer patients with nodal stage N0 and N1: who could benefit from post-mastectomy radiotherapy? St-Pierre Y, ed. PLoS One 10(12) e0145463 https://doi.org/10.1371/journal.pone.0145463

39. Trovo M, Durofil E, and Polesel J, et al (2012) Locoregional failure in early-stage breast cancer patients treated with radical mastectomy and adjuvant systemic therapy: which patients benefit from postmastectomy irradiation? Int $J$ Radiat Oncol Biol Phys 83 e153-e157 https://doi.org/10.1016/j.jijrobp.2011.12.050 PMID: 22386375

40. Truong PT, Lesperance M, and Culhaci A, et al (2005) Patient subsets with T1-T2, node-negative breast cancer at high locoregional recurrence risk after mastectomy Int J Radiat Oncol Biol Phys 62 175-182 https://doi.org/10.1016/j.jirobp.2004.09.013 PMID: 15850919

41. Sharma R, Bedrosian I, and Lucci A, et al (2010) Present-day locoregional control in patients with $\mathrm{t} 1$ or $\mathrm{t} 2$ breast cancer with 0 and 1 to 3 positive lymph nodes after mastectomy without radiotherapy Ann Surg Oncol 17 2899-2908 https://doi.org/10.1245/ s10434-010-1089-x PMID: 20443145 PMCID: 4324592

42. Rowell NP (2010) Are mastectomy resection margins of clinical relevance? A systematic review Breast 19 14-22 https://doi. org/10.1016/j.breast.2009.10.007

43. Hastings J, Iganej S, and Huang C, et al (2014) Risk factors for locoregional recurrence after mastectomy in stage T1 N0 breast cancer Am J Clin Oncol 37(5) 486-491 https://doi.org/10.1097/COC.0b013e31827e54c2

44. Lehr HA, Hansen DA, and Kussick S, et al (1999) Assess- ment of proliferative activity in breast cancer: MIB-1 immunohistochemistry versus mitotic figure count Hum Pathol 30 1314-1320 https://doi.org/10.1016/S0046-8177(99)90062-X PMID: 10571511

45. Thor AD, Liu S, and Moore DH, et al (1999) Compari- son of mitotic index, in vitro bromodeoxyuridine labeling, and MIB-1 assays to quantitate proliferation in breast cancer J Clin Oncol 17 470-477 https://doi.org/10.1200/JCO.1999.17.2.470 PMID: 10080587

46. de Azambuja E, Cardoso F, and de CG Jr, et al (2007) Ki-67 as prognostic marker in early breast cancer: a meta-analysis of published studies involving 12155 patients Br J Cancer 96 1504-1513 https://doi.org/10.1038/sj.bjc.6603756 PMID: 17453008 PMCID: 2359936

47. Selz J, Stevens D, and Jouanneau L, et al (2012) Prognostic value of molecular subtypes, ki67 expression and impact of postmastectomy radiation therapy in breast cancer patients with negative lymph nodes after mastectomy Int $J$ Radiat Oncol Biol Phys 84 1123-1132 https://doi.org/10.1016/j.jjrobp.2012.02.047 PMID: 22572073

48. Wang J, Shi M, and Ling R, et al (2011) Adjuvant chemotherapy and radiotherapy in triple-negative breast carcinoma: a prospective randomized controlled multi-center trial Radiother Oncol 100 200-204 https://doi.org/10.1016/..radonc.2011.07.007 PMID: $\underline{21852010}$

49. Nielsen HM, Overgaard M, and Grau C, et al (2006) Study of failure pattern among high-risk breast cancer patients with or without postmastectomy radiotherapy in addition to adjuvant systemic therapy: long-term results from the danish breast cancer cooperative group DBCG 82 b and c Randomized Studies J Clin Oncol 24(15) 2268-2275 https://doi.org/10.1200/ $\underline{\text { JCO.2005.02.8738 PMID: } 16618947}$

50. Helinto M, Blomqvist C, and Heikkila P, et al (1999) Post-mastectomy radiotherapy in pT3NOMO breast cancer: is it needed? Radiother Oncol 52 213-217 https://doi.org/10.1016/S0167-8140(99)00099-7 PMID: 10580866

51. Floyd SR, Buchholz TA, and Haffty BG, et al (2006) Low local recurrence rate without postmastectomy radiation in nodenegative breast cancer patients with tumors $\mathbf{5}$ cm and larger Int J Radiat Oncol Biol Phys 66 358-364 https://doi.org/10.1016/j. ijrobp.2006.05.001 PMID: 16887288

52. Taghian AG, Jeong JH, and Mamounas EP, et al (2006) Low locoregional recurrence rate among node-negative breast cancer patients with tumors $5 \mathrm{~cm}$ or larger treated by mastectomy, with or without adjuvant systemic therapy and without radiotherapy: results from five national surgical adjuvant breast and bowel project randomized clinical trials J Clin Oncol 24 3927-3932 https://doi.org/10.1200/JCO.2006.06.9054 PMID: 16921044 
53. Goulart J, Truong P, and Woods R, et al (2011) Outcomes of node-negative breast cancer 5 centimeters and larger treated with and without postmastectomy radiotherapy Int J Radiat Oncol Biol Phys 80 758-764 https://doi.org/10.1016/j.jirobp.2010.02.014

54. Mignano JE, Gage I, and Piantadosi S, et al (2007) Local recurrence after mastectomy in patients with T3pNO breast carcinoma treated without postoperative radiation therapy Am J Clin Oncol 30 466-472 https://doi.org/10.1097/COC.0b013e31805c13ba PMID: 17921705

55. McCammon R, Finlayson C, and Schwer A, et al (2008) Impact of postmastectomy radiotherapy in T3NO invasive carcinoma of the breast: a Surveillance, Epidemiology, and End Results database analysis Cancer 113 683-689 https://doi.org/10.1002/ cncr.23611 PMID: 18543316

56. Johnson ME, Handorf EA, and Martin JM, et al (2014) Post-mastectomy radiation therapy for T3N0: a SEER analysis Cancer 120(22) 3569-3574 https://doi.org/10.1002/cncr.28865 PMID: 24985911 PMCID: 4413466

57. Albert JM, Gonzalez-Angulo AM, and Guray M, et al (2010) Estrogen/progesterone receptor negativity and HER2 positivity predict locoregional recurrence in patients with T1a,bN0 breast cancer Int J Radiat Oncol Biol Phys 77 1296-1302 https://doi. org/10.1016/j.jijrobp.2009.12.011 PMID: 20472353

58. Adkins FC, Gonzalez-Angulo AM, and Lei X, et al (2011) Triple-negative breast cancer is not a contraindication for breast conservation Ann Surg Oncol 18 3164-3173 https://doi.org/10.1245/s10434-011-1920-z PMID: 21947595 PMCID: 4337779

59. Zumsteg ZS, Morrow M, and Arnold B, et al (2013) Breast-conserving therapy achieves locoregional outcomes comparable to mastectomy in women with T1-2N0 triple-negative breast cancer Ann Surg Oncol 20 3469-3476 https://doi.org/10.1245/s10434013-3011-9 PMID: 23686101 PMCID: $\underline{5730455}$

60. Fitzgibbons PL, et al (2000) Prognostic factors in breast cancer. College of American Pathologists Consensus Statement 1999 Arch Pathol Lab Med 124 966-978 PMID: 10888772

61. Lee AH, Pinder SE, and Macmillan RD, et al (2006) Prognostic value of lymphovascular invasion in women with lymph node negative invasive breast carcinoma Eur J Cancer 42 357-362 https://doi.org/10.1016/i.eica.2005.10.021

62. Boutrus R, Abi-Raad R, and Niemierko A, et al (2010) Does lymphovascular invasion predict regional nodal failure in breast cancer patients with zero to three positive lymph nodes treated with conserving surgery and radiotherapy? Implications for regional radiation Int J Radiat Oncol Biol Phys 78 793-798 https://doi.org/10.1016/j.jirobp.2009.08.049 PMID: 20171799

63. Wallgren A, Bonetti M, and Gelber RD, et al (2003) Risk factors for locoregional recurrence among breast cancer patients: results from International Breast Cancer Study Group Trials I through VII J Clin Oncol 21 1205-1213 https://doi.org/10.1200/ JCO.2003.03.130 PMID: 12663706

64. Ejlertsen B, Jensen MB, and Rank F, et al (2009) Population-based study of peritumorallymphovascular invasion and outcome among patients with operable breast cancer J Natl Cancer Inst 101 729-735 https://doi.org/10.1093/jnci/djp090 PMID: 19436035

65. Carter CL, Allen C, and Henson DE (1989) Relation of tumor size, lymph node status, and survival in 24,740 breast cancer cases Cancer 63 181-187 PMID: 2910416

66. Yamazaki N, Wada N, and Yamauchi C, et al (2015) High expression of post-treatment Ki-67 status is a risk factor for locoregional recurrence following breast-conserving surgery after neoadjuvant chemotherapy Eur J Surg Oncol 41, 617-624 https:// doi.org/10.1016/j.ejso.2015.01.036 PMID: 25771264

67. Synnestvedt M, Borgen E, and Russnes HG, et al (2013) Combined analysis of vascular invasion, grade, HER2 and Ki67 expression identifies early breast cancer patients with questionable benefit of systemic adjuvant therapy Acta Oncol 52 91-101 https://doi.org/10.3109/0284186X.2012.713508

68. Kilickap S, Kaya Y, and Yucel B, et al (2014) Higher Ki67 expression is associates with unfavorable prognostic factors and shorter survival in breast cancer Asian Pac J Cancer Prev 15 1381-1385 https://doi.org/10.7314/APJCP.2014.15.3.1381 PMID: $\underline{24606469}$ 
69. Thomas JS, Hanby AM, and Russell N, et al (2017) The BIG 2.04 MRC/EORTC SUPREMO Trial: pathology quality assurance of a large phase 3 randomised international clinical trial of postmastectomy radiotherapy in intermediate-risk breast cáncer Breast Cancer Res Treatment 163(1) 63-69 https://doi.org/10.1007/s10549-017-4145-4

70. Aalders KC, Postma EL, and Strobbe LJ, et al (2016) Contemporary locoregional recurrence rates in young patients with early-stage breast cancer J Clin Oncol 34(18) 2107-2114 PMID: 26976422

71. Azim HA Jr, Michiels S, and Bedard PL, et al (2012) Elucidating prognosis and biology of breast cancer arising in young women using gene expression profiling Clin Cancer Res 18 1341-1351 https://doi.org/10.1158/1078-0432.CCR-11-2599 PMID: 22261811

72. Elkhuizen PH, van de Vijver MJ, and Hermans J, et al (1998) Local recurrence after breast-conserving therapy for invasive breast cancer: high incidence in young patients and association with poor survival Int J Radiat Oncol Biol Phys $40859-867$ https://doi. org/10.1016/S0360-3016(97)00917-6 PMID: 9531371 